\title{
Caracterização morfológica de Alternaria alternata (Fr.:Fr.) Keissl. ocorrente em solanáceas ${ }^{1}$
}

\author{
Paulo Henrique Pereira Costa Muniz ${ }^{2}$; Jessica Borges de Oliveira ${ }^{3}$; Gustavo Henrique Silva \\ Peixoto $^{4}$; Fabricio Rodrigues ${ }^{5}$; Daniel Diego Costa Carvalho ${ }^{6}$
}

Resumo: A pinta preta, cujo agente causal é o fungo Alternaria spp., encontra-se distribuída pelas áreas de plantio de solanáceas, registrando consideráveis perdas de produção em diversas regiões do mundo. Neste sentido, a correta identificação do patógeno consiste no primeiro passo para o manejo da doença, visando a garantia do estabelecimento uniforme das culturas em campo. Objetivou-se com este trabalho caracterizar o fungo Alternaria sp. ocorrente em órgãos vegetativos de tomate e batateira. Em inspeções fitossanitárias em propriedades rurais do município de Ipameri, Goiás, foram coletadas duas amostras de folhas de tomateiro, uma de fruto e uma de folhas de batateira exibindo lesões, as quais foram examinadas em estereomicroscópio. Para caracterização micromorfológica, foram confeccionadas lâminas semipermanentes, mediante a remoção de micélio e estruturas fúngicas encontradas sobre o tecido vegetal infectado. A espécie encontrada nos materiais de solanáceas analisados apresentou conídios com 18,4 - 54,8 x 5,8 - 18,4 $\mu \mathrm{m}$, formato de pera invertida, coloração marrom clara e bico de 3,2-17,2 $\mu \mathrm{m}$. Portanto, verifica-se a ocorrência da espécie A. alternata para as quatro amostras analisadas.

Palavras-chave: Horticultura, Micologia; Taxonomia de fungos; Solanum tuberosum; Solanum lycopersicum.

\section{Morphological characterization of Alternaria alternata (Fr.:Fr.) Keissl. occurring in solanaceae}

\begin{abstract}
The black point, which causal agent is the fungus Alternaria spp., is distributed in the solanaceous planting areas, registering considerable losses of production in several regions of the world. In this sense, the correct identification of the pathogen is the first step in the disease management, aiming to guarantee an uniform establishment of the crops in the field. The objective of this work was to characterize Alternaria sp. fungus, occurring in vegetative organs of tomato and potato. In phytosanitary inspections on rural properties in the municipality of Ipameri, Goiás, two samples of tomato leaves, one of fruit and one of potato leaves showing lesions were collected, which were examined in stereomicroscope. For micromorphological characterization, semipermanent slides mountings were made by removing mycelium and fungal structures found on infected plant tissue. The species found in the analyzed solanaceous materials showed conidia with $18.4-54.8$ x $5.8-18.4 \mu \mathrm{m}$, inverted pear shape, light brown coloration and a beak of $3.2-17.2 \mu \mathrm{m}$. Therefore, it is verified A. alternata occurrence on the four samples analyzed.
\end{abstract}

Key-words: Horticulture; Mycology; Fungal taxonomy; Solanum tuberosum; Solanum lycopersicum.

\footnotetext{
${ }^{1}$ Submetido em 15/08/2017 e aprovado em 22/02/2018

${ }^{2}$ Graduando em Agronomia, Universidade Estadual de Goiás (UEG), Laboratório de Fitopatologia, Ipameri-GO, CEP: 75.780-000; E-mail: paulohenrique.muniz1@gmail.com

${ }^{3}$ Mestre em Produção Vegetal, Universidade Estadual de Goiás (UEG), Laboratório de Fitopatologia, Ipameri-GO, CEP: 75.780-000; E-mail: jessicaborges114@hotmail.com

${ }^{4}$ Graduando em Agronomia, Universidade Estadual de Goiás (UEG), Laboratório de Fitopatologia, Ipameri-GO, CEP: 75.780-000; E-mail: gugspeixoto@gmail.com

${ }^{5}$ Doutor em Genética e Melhoramento de Plantas; Professor, Universidade Estadual de Goiás (UEG), Programa de Pós-Graduação em Produção Vegetal, Ipameri-GO, CEP: 75.780-000; E-mail: fabricio.rodrigues@ueg.br

${ }^{6}$ Doutor em Fitopatologia; Professor, Universidade Estadual de Goiás (UEG), Laboratório de Fitopatologia, Ipameri-GO, CEP: 75.780-000; Email: daniel.carvalho@ueg.br
} 


\section{Introdução}

Originárias dos Andes e consideradas entre as quatro principais espécies cultivadas, a batata (Solanum tuberosum L.) e o tomate (Solanum lycopersicum L.) constituem a base da alimentação da população de diversos países e culturas (Fiers et al. 2010). As condições favoráveis de clima, solo e demanda pelo produto, fazem do Brasil um grande produtor de tomate $\mathrm{e}$ batata. A produção brasileira de tomate em 2016, alcançou 3544,6 mil toneladas produzidos em uma área de 54,7 mil ha (IBGE, 2016). Em consonância, a produção de batata estimada para 2017 é de 3,9 milhões de toneladas, produzidas em uma área de 130 mil ha, reafirmando-a como a hortaliça mais importante no país (IBGE, 2017).

Em consequência da crescente expansão do plantio das solanáceas, a batateira e tomateiro tem se tornado hospedeiros de inúmeros patógenos (Töfoli et al. 2013; Inoue-Nagata et al., 2016). Neste contexto, a pinta preta, causada pelo fungo Alternaria spp., um importante fungo patogênico, consiste em uma das principais doenças que ocasionam danos às solanáceas (Dias et al., 2016). Decorrente de regiões tropicais, a doença registra consideráveis perdas de produção em diversas regiões do mundo, ocasionando decréscimos que atingem a ordem de até $60 \%$ de tubérculos de batata (Miles et al., 2013) e em geral, apresenta até $30 \%$ de danos na produtividade total em regiões produtoras (Yanar et al., 2011). No tomate as perdas em decorrência do ataque do fungo podem atingir 100\% da produção, caso não sejam empregadas medidas de controle (TöfolI et al., 2014).

Segundo Boiteux e Reifschneider (1994) a A. solani é o principal agente causal da pinta preta da batata, no Brasil. Embora, tenha sido descrito, em diversos relatos, como o fungo agente causal da pinta preta (Yanar et al., 2011; Leiminger e Hausladen, 2012), outras espécies do gênero podem ser considerados agentes causais da doença em batata e tomate, como A. alternata (Cwalina-Ambroziak e Bogucka, 2012; Vasconcelos et al., 2014), A. grandis, observadas em Goiás e Minas Gerais (Rodrigues et al., 2010), e restritamente à tomate, A. tomatophila e A. cretica ocorrente nos estados de Minas Gerais e São Paulo (Töfoli et al., 2014).

De modo geral, as diferenças sintomatológicas entre as espécies do gênero Alternaria não são observadas, mas estas podem ser diferenciadas por meio da morfologia dos conídios (Rodrigues et al., 2010; Töfoli et al., 2013; Vasconcelos et al., 2014). No caso específico do gênero Alternaria, que ocorre em diversos hospedeiros, as características morfológicas mais importantes para a caracterização e a consequente identificação das espécies são as dimensões dos conídios e dos bicos dos conídios que cada uma apresenta (Rodrigues et al., 2010; Alhussaen, 2012; Vasconcelos et al., 2014).

Neste contexto, a correta identificação do patógeno consiste no primeiro passo em direção ao posterior manejo da doença por ele causada (Carvalho et al., 2014). Diante disso, em observância à grande importância que as espécies da família Solanaceae representam para a segurança alimentar mundial e pelas grandes perdas registradas por conta da ação patogênica de Alternaria spp., faz-se necessária a caracterização de espécies desse gênero observando-se caracteres micromorfológicos. Objetivou-se com este trabalho caracterizar morfologicamente o fungo Alternaria, proveniente da parte vegetativa, de tomate e de batata e fruto de tomate.

\section{Material e Métodos}

\subsection{Levantamento de ocorrência de alternariose em campo}

Em inspeções de campo, durante os meses de novembro de 2016 e maio de 2017, foram realizadas avaliações fitossanitárias em propriedades agrícolas do município de Ipameri e região. Segundo a classificação de Köppen esta região possui clima tropical com inverno seco e verão úmido $(\mathrm{Aw})$, com temperatura média de $23,1^{\circ} \mathrm{C}$ e pluviosidade anual média de $1437 \mathrm{~mm}$. Durante o período do experimento (meses de novembro de 2016 e maio de 2017), as temperaturas máxima e mínima nos locais de amostragens, apresentou valores variando de 17 a $33^{\circ} \mathrm{C}$ e 11 a $30^{\circ} \mathrm{C}$, respectivamente e precipitação de 242,1 e $41,9 \mathrm{~mm}$, respectivamente.

As avaliações em campo englobaram os quadros sintomatológicos, aspectos epidemiológicos e edafoclimáticos nas culturas do tomateiro. Adicionalmente, frutos de tomateiro obtidos do comércio local também foram inspecionados. Em seguida, os materiais

Revista Agropecuária Técnica, Areia-PB, v. 39, n. 1, p. 43-49, 2018

DOI: 10.25066/agrotec.v39i1. 35699 
foram coletados para exame no Laboratório de Fitopatologia da Universidade Estadual de Goiás (UEG), Câmpus Ipameri. Após as inspeções, caracterização de sintomas e confecção de lâminas, quatro materiais foram obtidos para análise: B-01-01 (conídios de Alternaria spp. ocorrente em $S$. tuberosum/folhas cv. 'Asterix', local de coleta: $17^{\circ} 43^{\prime} 00.38^{\prime}$ 'S, $48^{\circ} 08^{\prime} 40.96^{\prime \prime} \mathrm{W}$, $796 \mathrm{~m}$, data de coleta: 1\%11/2016); J-01-01 (ocorrente em S. lycopersicum/folhas cv. 'Santa Cruz Kada', local de coleta: 17 40 '18.48”'S, $47^{\circ} 44^{\prime} 57.22^{\prime \prime} \mathrm{W}, 905 \mathrm{~m}$, data de coleta: 02/11/2016) e J-01-02 (ocorrente em $S$. lycopersicum/folhas cv. 'Santa Clara', local de coleta: $17^{\circ} 40^{\prime} 18.48^{\prime}$ 'S, $\left.47^{\circ} 44^{\prime} 57.22^{\prime \prime} \mathrm{W}, 905 \mathrm{~m}\right) \mathrm{e}$ J-01-03 (ocorrente em $S$. lycopersicum/fruto cv. 'Débora', local de coleta: 17\%43'18.26"S, 4809'40.61”'W, $766 \mathrm{~m}$ ).

As lesões de tecidos vegetais atacados foram examinadas em estereomicroscópio, fotografadas e as que apresentaram micélio em abundância foram empregadas para confecção de lâminas microscópicas semipermanentes.

\subsection{Caracterização micromorfológica}

Para caracterização morfológica e confirmação do gênero fúngico incidente no material, foram confeccionadas lâminas semipermanentes para observações microscópicas, contendo o material biológico removido diretamente do tecido vegetal infectado e utilizando ácido lático como meio de montagem para as lâminas (Lima et al., 2004). A caracterização morfológica foi baseada nas seguintes características: comprimento e largura dos conídios e, comprimento do bico dos conídios. Para tanto, foi realizado o registro de imagens e mensuração dos conídios e suas estruturas, pela obtenção de 30 medidas de cada componente (Vasconcelos et al., 2014), no microscópio de luz Leica DM500, com auxílio do programa LAS EZ $2.0(100 \mathrm{x})$. As preparações microscópicas foram depositadas no Laminário Fitopatológico da UEG.

\section{Resultados e Discussão}

Segundo Töfoli et al. (2014), a ocorrência da pinta preta em solanáceas é favorecida por temperaturas na faixa de 22 a $32^{\circ} \mathrm{C}$, elevada umidade e alternâncias de períodos secos e úmidos. Durantes os meses de novembro de 2016 e maio de 2017, a temperatura no município de Ipameri, apresentou valores variando de 17 a $33^{\circ}$ C e 11 a $30^{\circ} \mathrm{C}$, respectivamente, condição que, associada à precipitação de 242,1 e $41,9 \mathrm{~mm}$, respectivamente, favoreceu o desenvolvimento da doença. Isto demonstra que a doença apresenta maior severidade em verões chuvosos, mas também pode ocorrer no inverno.

Os conídios obtidos do material B-01-01 apresentaram dimensões de 25,3 - 54,8 x 6,1 $18,4 \mu \mathrm{m}(38,3 \times 12,3 \mu \mathrm{m})$ e bico de 5,0 - 17,2 $\mu \mathrm{m}$ $(8,8 \mu \mathrm{m})$. Após a mensuração dos conídios do material J-01-01, obtido de folhas de tomateiro cv. Santa Cruz Kada lesionadas, verificou-se que estes possuíam dimensões de 20,5 - 31,0 x 6,4 $12,3 \mu \mathrm{m}(25,5 \times 9,0 \mu \mathrm{m})$ e bico do conídio 3,2 $6,9 \mu \mathrm{m}(4,9 \mu \mathrm{m})$. Para J-01-02 as medidas foram $18,5-42,0 \times 6,0-18,1 \mu \mathrm{m}(30,2 \times 10,2 \mu \mathrm{m}) \mathrm{e}$ bico de 4,0-12,4 $\mu \mathrm{m}(6,6 \mu \mathrm{m})$ e para J-01-03, as medidas de $18,4-34,5 \times 5,8-13,0 \mu \mathrm{m}(25,3 \times$ $8,1 \mu \mathrm{m})$ e bico de $3,6-11,6 \mu \mathrm{m}(6,0 \mu \mathrm{m})$ (Tabela 1). Os conídios de todos os materiais analisados apresentaram coloração marrom clara, em forma de clava ou pera invertida (Figura 1). Além disso, o bico dos conídios de B-01-01, J-01-01, J-01-02 e J-01-03 foi considerado como de tamanho curto, com comprimento inferior a um terço do corpo do conídio.

Tabela 1 Características micromorfológicas de conídios de Alternaria spp. ocorrente em folhas e fruto de tomateiro, e folhas de batateira.

\begin{tabular}{|c|c|c|c|c|}
\hline \multirow{2}{*}{$\begin{array}{l}\text { Código do material } \\
\text { analisado }\end{array}$} & Hospedeiro/ & \multicolumn{2}{|c|}{ Conídio $(\mu \mathrm{m})$} & Bico do conídio \\
\hline & & Comprimento & Largura & Comprimento \\
\hline B-01-01 & S. tuberosum/folhas ${ }^{1}$ & $25,3-54,8$ & $6,1-18,4$ & $5,0-17,2$ \\
\hline $\mathrm{J}-01-01$ & S. lycopersicum/folhas ${ }^{2}$ & $20,5-31,0$ & $6,4-12,3$ & $3,2-6,9$ \\
\hline $\mathrm{J}-01-02$ & S. lycopersicum/folhas 3 & $18,5-42,0$ & $6,0-18,1$ & $4,0-12,4$ \\
\hline $\mathrm{J}-01-03$ & S. lycopersicum/fruto ${ }^{4}$ & $18,4-34,5$ & $5,8-13,0$ & $3,6-11,6$ \\
\hline
\end{tabular}

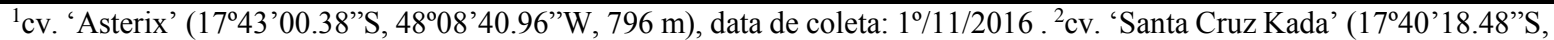

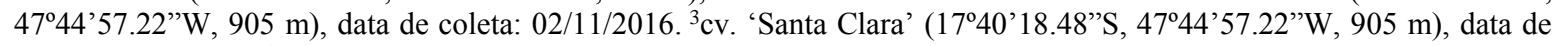
coleta: 21/11/2016. ${ }^{4} \mathrm{cv}$. 'Débora' (1743'18.26”S, 4809'40.61”W, $\left.766 \mathrm{~m}\right)$, data de coleta: 25/05/2017.

Revista Agropecuária Técnica, Areia-PB, v. 39, n. 1, p. 43-49, 2018

DOI: 10.25066/agrotec.v39i1. 35699 

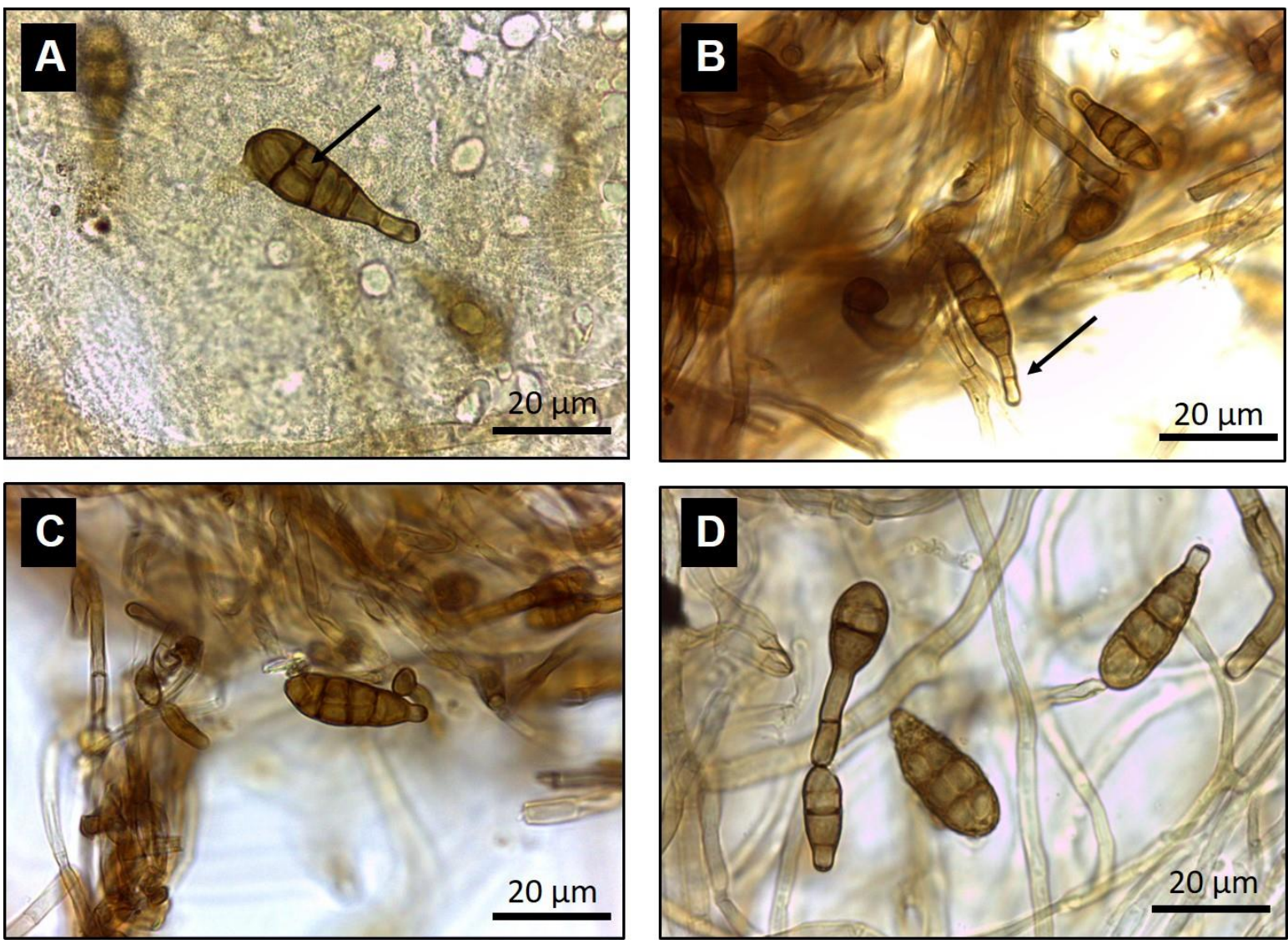

Figura 1 Conídios de Alternaria alternata provenientes de solanáceas. A) conídio de B-01-01 de coloração marrom clara, em forma de clava ou pera invertida ovoides ou elipsoides, a seta mostra os septos longitudinais e transversais. B) conídio de J-01-01, a seta mostra o bico curto, com comprimento inferior a um terço do corpo do conídio. C) Conídio de J-01-02. D) Conídio de J-01-03. 100 x. Ipameri, Goiás, 2017.

O sinal do patógeno em lesões incidentes diretamente no órgão das plantas analisadas, apresentaram conformidade com as descritas por Zheng et al. (2015), que relatou a produção de um micélio de coloração cinza escuro a preto, aéreo, densamente turfado, com produção de conidióforos simples com conídios escuros e multiseptais para A. alternata.

Adicionalmente, para as comparações e determinações à nível de espécie, foram utilizadas as características morfológicas de outras espécies do gênero Alternaria ocorrentes nas solanáceas e A. alternata ocorrentes em hospedeiros diferentes. A Tabela 2 resume as características micromorfológicas de diferentes espécies do gênero Alternaria spp. ocorrentes e relatadas em culturas de importância econômica.

A espécie encontrada nos materiais de solanáceas analisados apresentou conídios com $18,4-54,8 \times 5,8-18,4 \mu \mathrm{m}$ e bico de $3,2-17,2$ $\mu \mathrm{m}$ (Tabela 1). Estas medidas sugerem a espécie tratar-se de A. alternata, uma vez que, em conformidade com a Tabela 2, as medições para A. alternata é verificada entre $18,0-60,0 \times 7,0-$ 20,0 $\mu \mathrm{m}$ e bico com 2,0 - 40,0 $\mu \mathrm{m}$ (Grogan et al., 1975; Misaghi et al., 1978; Van der Walls et al., 2011; Vasconcelos et al., 2014).

De forma oposta, os resultados das dimensões dos conídios de A. grandis (102,0 - 184,0 x 14,0 - 17,7 $\mu \mathrm{m})$ e dos bicos dos conídios $(120,0-$ $206,0 \mu \mathrm{m})$ verificados por Rodrigues et al. (2010) e Bessadat et al., (2016), em folhas de batata e tomateiro, respectivamente, são muito diferentes das medições reportadas para A. alternata, especialmente com respeito ao comprimento dos conídios, bem como ao bico dos conídios. De forma análoga, embora a espécie A. solani seja considerada o principal agente causal da pinta preta nas solanáceas, possuindo similaridades, em relação à forma e coloração dos conídios, sua ocorrência pode ser refutada, uma vez que os conídios dessa espécie possuem maior 
comprimento, isto é, $82,5-159,5 \mu \mathrm{m}$, possuindo bicos do conídio também muito maiores $47,0-$
$86,0 \mu \mathrm{m}$ (Zheng et al., 2015; Bessadat et al., 2017).

Tabela 2 Características morfológicas de diferentes espécies do gênero Alternaria, em diferentes hospedeiros

\begin{tabular}{|c|c|c|c|c|}
\hline \multirow{2}{*}{$\begin{array}{l}\text { Espécie de } \\
\text { Alternaria }\end{array}$} & \multirow{2}{*}{$\begin{array}{c}\text { Hospedeiro / órgão da } \\
\text { planta }\end{array}$} & \multicolumn{2}{|c|}{ Tamanho do conídio $(\mu \mathrm{m})$} & \multirow{2}{*}{$\begin{array}{l}\text { Tamanho do bico } \\
(\boldsymbol{\mu m})\end{array}$} \\
\hline & & Comprimento & Largura & \\
\hline A. grandis $^{(1)}$ & S. tuberosum / folha & $102,0-184,0$ & $14,0-17,0$ & $135,0-206,0$ \\
\hline A. grandis (2) & S. lycopersicum / folha & $140,9-158,7$ & $15,1-17,7$ & 120,0 \\
\hline A. solani ${ }^{(3)}$ & S. tuberosum / folha & $82,5-105,0$ & $16,7-20,5$ & $60,0-84,7$ \\
\hline A. solani ${ }^{(4)}$ & S. lycopersicum / fruto & $115,2-159,5$ & $15,0-17,9$ & $47,0-86,0$ \\
\hline A. tomatophila ${ }^{(1)}$ & S. lycopersicum / folha & $70,0-99,0$ & $12,0-20,0$ & $99,0-197,0$ \\
\hline A. cretica $^{(5)}$ & S. lycopersicum / folha & $83,0-110,0$ & $14,0-17,0$ & $160,0-250,0$ \\
\hline${\text { A. } \text { alternata }^{(6)}}^{(6)}$ & S. tuberosum / folha & $20,0-60,0$ & $9,0-18,0$ & $*$ \\
\hline A. alternata $^{(7)}$ & S. tuberosum / tubérc. & $19,7-45,4$ & $8,5-18,0$ & $2,6-11,9$ \\
\hline A. alternata $^{(8)}$ & S. lycopersicum / caule & $18,0-50,0$ & $7,0-18,0$ & $2,0-20,0$ \\
\hline A. alternata $^{(9)}$ & S. lycopersicum / fruto & $18,0-55,0$ & $7,0-20,0$ & $5,0-40,0$ \\
\hline
\end{tabular}

${ }^{(1)}$ Rodrigues et al., (2010); (2)Bessadat et al., (2016); ${ }^{(3)}$ Zheng et al., (2015); ${ }^{(4)}$ Bessadat et al., (2017); ${ }^{(5)}$ Simmons, (2000);

${ }^{(6)}$ Van der Walls et al., (2011); ${ }^{(7)}$ Vasconcelos et al., (2014); ${ }^{(8)}$ Misaghi et al., (1978); ${ }^{(9)}$ Grogan et al., (1975).

A hipótese de a espécie a que pertence os materiais B-01-01, J-01-01, J-01-02 e J-01-03 ser A. tomatophila e A. cretica também pode ser refutada. Embora seja reportada a ocorrência dessas espécies em folha de $S$. lycopersicum (Simmons, 2000; Rodrigues et al., 2010), o comprimento dos conídios de A. tomatophila e A. cretica $(70,0-99,0 \mu \mathrm{m}$ e $83,0-110,0 \mu \mathrm{m}$, respectivamente), bem como o tamanho dos bicos dos conídios $(99,0-197,0 \mu \mathrm{m}$ e 160,0 - 250,0 $\mu \mathrm{m}$, respectivamente) são muito grandes quando comparados com as medidas expostas na Tabela 1. Töfoli et al. (2013) já afirmaram que os bicos dos conídios de $A$. alternata são pequenos o suficiente, a ponto de seu comprimento ser inferior a um terço do comprimento do corpo do conídio (Figura 1B), fato que não ocorre para $A$. solani e A. grandis, A. tomatophila e A. cretica ocorrentes em S. tuberosum e S. lycopersicum.

\section{Conclusão}

Baseando-se nas características micromorfológicas dos conídios analisados, verificou-se tratar da ocorrência de A. alternata em folhas de tomateiro cv. 'Santa Cruz Kada' e 'Santa Clara'; em fruto de tomateiro cv. 'Débora' e em folhas de batateira cv. 'Asterix'.

\section{Agradecimentos}

Os autores agradecem ao Conselho Nacional de Desenvolvimento Científico e Tecnológico $(\mathrm{CNPq})$, por uma bolsa de iniciação científica concedida e ao Programa de Bolsa de Incentivo à Pesquisa e Produção Científica (PROBIP) da Universidade Estadual de Goiás (UEG) por duas bolsas de incentivo à pesquisa.

\section{Referências}

Alhussaen, K. M. Morphological and physiological characterization of Alternaria solani isolated from tomato in Jordan Valley. Research Journal of Biological Sciences, v.7, n.8, p.316-319, 2012. https://www.researchgate.net/publication/286290551_Mo rphological_and_Physiological_Characterization_of_Alte rnaria_solani_Isolated_from_Tomato_in_Jordan_Valley

Bessadat, N.; Berruyer, R.; Hamon, B.; BatailleSimoneau, N.; Benichou, S.; Kihal, M.; Henni, D. E.; Simoneau, P. Alternaria species associated with early blight epidemics on tomato and other Solanaceae crops in northwestern Algeria. European Journal of Plant Pathology, v.148, n.1, p.181-197, 2017. https://link.springer.com/article/10.1007\%2Fs10658-016$\underline{1081-9}$

Bessadat, N.; Berruyer, R; Hamon, B.; BatailleSimoneau, N.; Benichou, S.; Kihal, M.; Henni, D. E.; Simoneau, P. First report of tomato early blight caused by Alternaria grandis in Algeria. Plant disease, v.100, n.2, p.533, 2016. https://doi.org/10.1094/PDIS-05-15-0613-PDN

Boiteux, L. S.; Reifschneider, F. J. B. Potato early blight caused by Alternaria alternata in Brazil. Plant Disease, v.78, n.1, p.101, 1994. 
https://www.apsnet.org/publications/PlantDisease/BackIs sues/Documents/1994Abstracts/PD_78_0101D.htm

Carvalho, D. D. C.; Oliveira, A. M. E.; Lago, H. M. da S.; Rodrigues, F. Incidência de Bipolaris bicolor em sementes de sorgo granífero no Brasil. Revista Brasileira de Milho e Sorgo, v.13, n.2, p.240-247, 2014. http://dx.doi.org/10.18512/1980-6477/rbms.v13n2p240$\underline{247}$

Cwalina-Ambroziak, B.; Bogucka, B. Severityof late blight (Phytophthora infestans/Mont./de Bary) and early blight of potato (Alternaria solani Sorauer, A. alternata/Fr./Keissler) in three potato cultivars under differentiated soil and foliar fertilization. Journal of Elementology, v.17, n.3, p.379-388, 2012. http://yadda.icm.edu.pl/yadda/element/bwmeta1.element. agro-ccfbb2c3-8520-41ed-b915-43ef6a714d84

Dias, J. A. C. S.; Iamati, M. T.; Fischer, I. H. Doenças da batateira. In: KimatI, H.; Amorim, L.; Rezende, J.A.M.; Bergamin Filho, A.; Camargo, L. E. A. Manual de Fitopatologia: Doenças das plantas cultivadas. 5 ed. Ouro Fino: Agronômica Ceres, v.2, 2016, p.125-148.

Ferreira, D.F. Sisvar: a computer statistical analysis system. Ciência e Agrotecnologia, Lavras, v.35, n.6, p.1039-1042, 2011. http://dx.doi.org/10.1590/S1413-70542011000600001

Grogan, R.G.; Kimble, K.A.; Misaghi, I. A steam canker of tomato caused by Alternaria alternata f.sp. lycopersici. Phytopathology, v.65, n.1, p.880-886,

1975.

https://www.apsnet.org/publications/phytopathology/back issues/Documents/1975Articles/Phyto65n08 880.PDF

IBGE - Instituto Brasileiro de Geografia e Estatística. Indicadores de produção agrícola para o ano de 2017. 2017. 72p. Disponível em: <ftp://ftp.ibge.gov.br/Producao Agricola/Fasciculo Indic adores_IBGE/estProdAgr_201701.pdf $>$ Acesso em: 04 jul. 2017.

IBGE - Instituto Brasileiro de Geografia e Estatística. Levantamento sistemático da produção agrícola: pesquisa mensal de acompanhamento das safras agrícolas no ano civil. 2016. Disponível em: <ftp://ftp.ibge.gov.br/Producao_Agricola/

Levantamento_Sistematico

_da_Producao_Agricola_[mensal]/Fasciculo/lspa_201603 .pdf> Acesso em: 04 jul. 2017.

Inoue-Nagata, A. K.; Lopes, C.A.; Reis, A.; Pereira, R.B.; Quezado-Duval, A.M.; Pinheiro,
J.B.; LIMA, F.M. Doenças do tomateiro. In: Kimati, H; Amorim, L.; Bergamin Filho, A.; Camargo, L. E. A.; Rezende, J. A. M. Manual de Fitopatologia: Doenças das plantas cultivadas. 5 ed. Ouro Fino: Agronômica Ceres, v.2, 2016, p.697-732.

Leiminger, J. H.; Hausladen, H. Early blight control in potato using disease-orientated threshold values. Plant Disease, v.96, n.1, p.124-130, 2012. http://dx.doi.org/10.1094/PDIS-05$11-0431$

Lima, C. S.; Souza, P. E.; Botelho, A. O. Rust fungi of the Pucciniaceae family on medicinal plants. Fitopatologia Brasileira, v.29, n.5, p. 99-503, 2004. http://dx.doi.org/10.1590/S0100$\underline{41582004000500005}$

Miles, T. D.; Miles, L. A.; Fairchild, K. L.; Wharton, P. S. Screening and characterization of resistance to succinate de hydrogenase inhibitors in Alternaria solani. Plant Pathology, v.63, n.1, p.155-164, 2013. https://doi.org/10.1111/ppa.12077

Misaghi, I. J.; Grogan, R. G.; Duniway, J. M.; Kimble, K. A. Influence of environment and culture Media on Spore Morphology of Alternaria alternata. Pytopatology, v.68, n.1, p.29-34,

1978. https://www.apsnet.org/publications/phytopathology/back issues/Documents/1978Articles/Phyto68n01_29.PDF

Rodrigues, T. T. M. S.; Berbee, M. L.; Simmons, E. G.; Cardoso, C. R.; Reis, A.; Maffia, L. A.; Mizubuti, E. S. G. First report of Alternaria tomatophila and $A$. grandis causing early blight on tomato and potato in Brazil. New Disease Reports, v.22, n.1, p. 28, 2010. http://dx.doi.org/10.5197/j.2044-0588.2010.022.028

Simmons, E. G. Alternaria themes and variations (244-286) species on Solanaceae. Mycotaxon, v.75, p.1-115, 2000. https://www.scienceopen.com/document?vid=086a210ee048-4d4b-96dc-109574982e0e

Töfoli, J. G.; Melo, P.C.T.; Domingues, R.J.; Ferrari, J.T. Potato late blight and early blight: importancy, characteristics and sustainable management. Biológico, v.75, n.1, p.33-40, 2013.

https://www.researchgate.net/publication/272176546_Re queima e pinta preta na cultura da batata importancia caracteristicas_e_manejo_sustentavel

Töfoli, J. G.; Melo, P. C. T.; Domingues, R. J.; Ferrari, J. T. Requeima e Mancha de Alternaria 
nas culturas da batata e tomate. Biológico, v.76, n.1, p.41-50, 2014. https://www.researchgate.net/publication/272481136_Re queima e mancha de alternaria nas culturas da batata e tomate

Van der Waals, J. E.; Pitsi, B. E.; Marais, C.; Wairuri, C. K. First report of Alternaria alternata causing leaf blight of potatoes in South Africa. Plant Disease, v.95, n.3, p.363, 2011. https://doi.org/10.1094/PDIS-11-10-0820

Vasconcelos, C. V.; Silva, D. C.; Carvalho, D. D. C. Ocorrência de Alternaria alternata (Fr.:Fr.) Keissl. em tubérculos de batata, no Brasil. Pesquisa Agropecuária Tropical, v.44, n.2, p.219-222, 2014. http://dx.doi.org/10.1590/S1983$\underline{40632014000200015}$

Yanar, Y.; Gökçe, I.; Kadioglu, I.; Çam, H.; Whalon, M. In vitro antifungal evaluation of various plant extracts against early blight disease (Alternaria solani) of potato. African Journal of Biotechnology v.10, n.42, p.82918295, 2011. http://dx.doi.org/10.5897/AJB11.241

Zheng, H.H.; Zhao, J.; Wang, T. Y.; Wu, X. H. Caracterization of Alternaria species associated with potato foliar diseases in China. Plant Pathology, v.64, n.2, p.425-433, 2015. https://doi.org/10.1111/ppa.12274 